\title{
LETTER
}

\section{Rapid detection of recurrent intraventricular hemorrhage by ultrasound in a multiple trauma patient who had undergone craniectomy}

\author{
Yu-Zhi Gao',2, Guang-Ju Zhou', Mao Zhang',*, Shou-Quan Chen³ and Jian-Xin Gan'
}

\begin{abstract}
Ultrasound may be a useful tool to evaluate intracranial abnormalities in critically ill patients undergoing decompressive craniectomy. We present a multiple trauma patient who had undergone craniectomy and in whom recurrent intraventricular hemorrhage and patterns of cerebral blood flow were rapidly detected by ultrasound.
\end{abstract}

Ultrasound has been widely used for rapid assessment of critical illness and is regarded as a visual stethoscope. There are few reports on the diagnosis of intracranial lesions by ultrasound in ICU patients. Here we present a multiple trauma patient who had undergone craniectomy and in whom recurrent intraventricular hemorrhage was rapidly detected by ultrasound.

A 29-year-old man was transferred from a local hospital 9 hours after a 5-meter fall. His Glascow Coma Score (GCS) was 4 and both pupils were unequal in size. The diagnosis was severe cerebral contusion and subdural hemotoma in the left temporal and parietal lobe, bilateral lung contusion and left clavicle fracture. He received an urgent decompressive craniectomy and evacuation of hematoma, and was then admitted to the emergency ICU. In the following days, his condition improved and his GCS reached 8, although this suddenly decreased to 6 on day 12. Bedside ultrasound was performed instantly and showed acute intraventricular hemorrhage (IVH), which was confirmed by subsequent computed tomography (CT) (Figure 1). Urgent bilateral ventricular external drainage was performed and his condition improved.

\footnotetext{
*Correspondence: zmhz@hotmail.com

'Department of Emergency Medicine, Second Affiliated Hospital, Zhejiang

University School of Medicine; Research Institute of Emergency Medicine Zhejiang

University, Hangzhou 310009, China

Full list of author information is available at the end of the article
}

Lumbar cistern tube drainage was performed on day 19. Unfortunately, he suddenly developed dilated pupils on day 20. Bedside ultrasound and a head CT scan were arranged immediately, both showing recurrence of IVH (Figure 1). The family refused further treatment and he then became brain dead (Figure 1).

Ultrasound is a useful tool to evaluate intracranial abnormalities in patients undergoing decompressive craniectomy. The absence of the skull may facilitate the ultrasound to detect the brain, and intracranial morphological abnormal signs such as dislocation of the midline, intracerebral hematomas, size of lateral ventricular and hydrocephalus may be clearly displayed on the screen [1,2]. This rapid and non-invasive method can complement the results of CT and may help to reduce delays in surgical management. Caricato and colleagues [3] found that ultrasound in patients with decompressive craniectomy was as effective as CT to evaluate intracerebral disorders. In this multiple trauma patient, IVH happened twice after the craniectomy and was immediately identified by ultrasound. Meanwhile, ultrasound may detect the characteristics of cerebral blood flow, which helps to assess intracranial pressure and cerebral perfusion pressure $[4,5]$. This highlights the special value of transcranial ultrasound in critically ill patients with craniectomy.

\section{Abbreviations \\ CT, computed tomography; GCS, Glascow Coma Score; IVH, intraventricular hemorrhage.}

\section{Competing interests}

The authors declare that they have no competing interests.

\section{Acknowledgements}

Written consent for publication was obtained from the patient's next of kin.

\section{Author details}

'Department of Emergency Medicine, Second Affiliated Hospital, Zhejiang University School of Medicine; Research Institute of Emergency Medicine Zhejiang University, Hangzhou 310009, China. ${ }^{2}$ Research fellow, from Department of Emergency Medicine, the First Affiliated Hospital of Wenzhou Medical College, Wenzhou 325000, China. ${ }^{3}$ Department of Emergency Medicine, the First Affiliated Hospital of Wenzhou Medical College, Wenzhou 325000, China. 

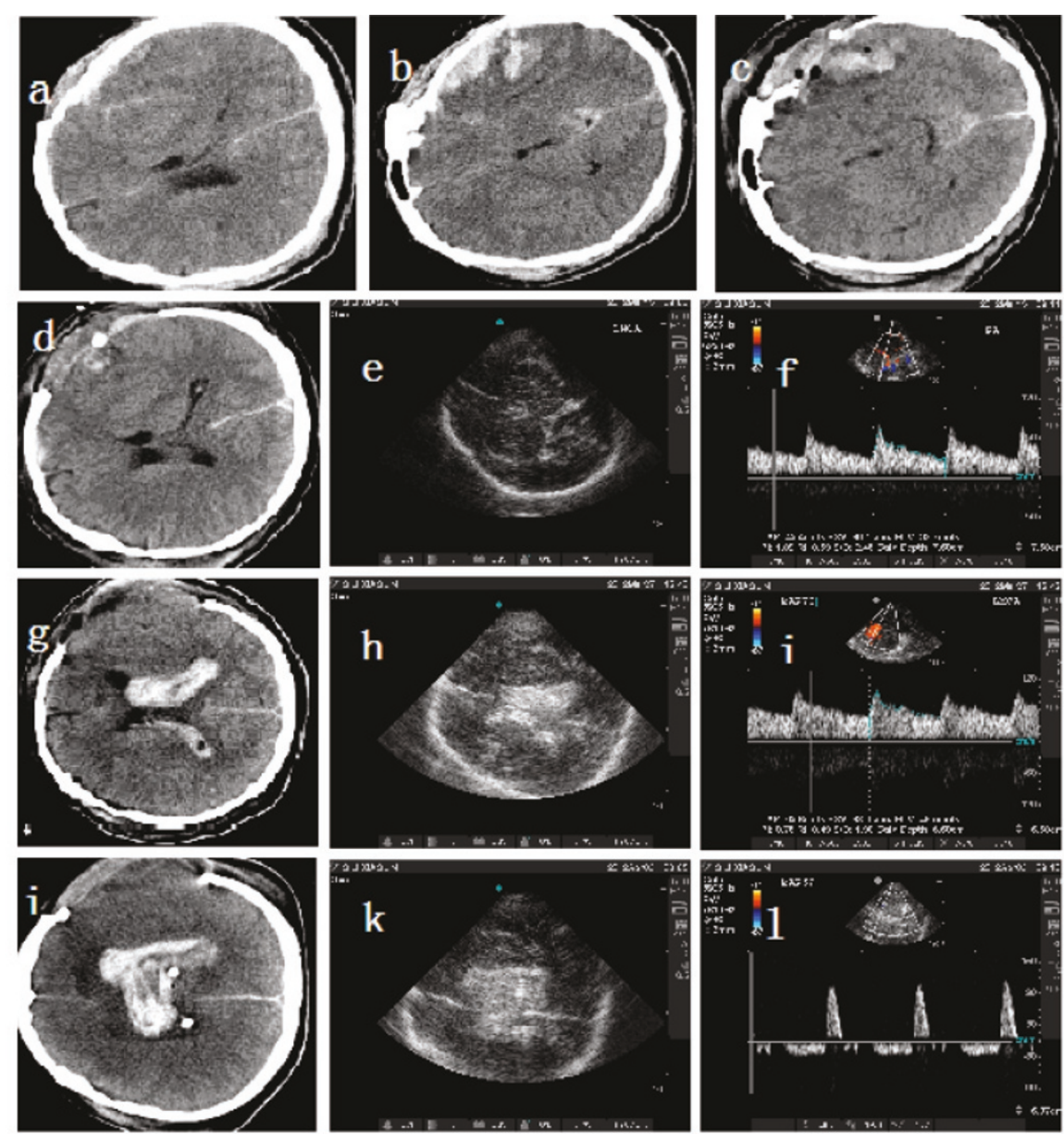

Figure 1. Computed tomography and ultrasound images of the head in a multiple trauma patient with craniectomy and recurrent intraventricular hemorrhage. (a,b) Head computed tomography (CT) before craniectomy showing severe cerebral contusion and the deformed left lateral ventricle. $(\mathbf{c}, \mathbf{d}) \mathrm{Head} \mathrm{CT}$ on the first day post-craniectomy showing the absence of the skull and the normal left lateral ventricle. (e) Ultrasound image corresponding to the CT scan shown in (d); (f) the normal Doppler pattern of the left middle cerebral artery (LMCA). (g-i) Head CT and ultrasound images after the first intraventricular hemorrhage (IVH): (g,h) hematocele in both lateral ventricles; (i) slightly abnormal Doppler pattern of the LMCA. (j-I) Head CT and ultrasound images after the second IVH: (j,k) both enlarged lateral ventricles with hematocele compared with $(\mathrm{g})$; (I) reverse blood flow during the diastolic period of LMCA.

\section{Published: 8 November 2012}

\section{References}

1. Ohue S, Kumon Y, Nagato S, Kohno S, Harada H, Nakagawa K, Kikuchi K, Miki $\mathrm{H}$, Ohnishi T: Evaluation of intraoperative brain shift using an ultrasoundlinked navigation system for brain tumor surgery. Neurol Med Chir (Tokyo) 2010, 50:291-300.

2. Behnke $S$, Becker $G$ : Sonographic imaging of the brain parenchyma. Eur J Ultrasound 2002, 16:73-80.

3. Caricato A, Mignani V, Bocci MG, Pennisi MA, Sandroni C, Tersali A, Antonaci A, de Waure C, Antonelli M: Usefulness of transcranial echography in patients with decompressive craniectomy: a comparison with computed tomography scan. Crit Care Med 2012, 40:1745-1752.
4. Lazaridis C: Advanced hemodynamic monitoring: principles and practice in neurocritical care. Neurocrit Care 2012, 16:163-169.

5. Brandi G, Bechir M, Sailer S, Haberthur C, Stocker R, Stover JF: Transcranial color-coded duplex sonography allows to assess cerebral perfusion pressure noninvasively following severe traumatic brain injury. Acta Neurochir (Wien) 2010, 152:965-972.

doi:10.1186/cc11509

Cite this article as: Gao YZ, et al.: Rapid detection of recurrent

intraventricular hemorrhage by ultrasound in a multiple trauma patient

who had undergone craniectomy. Critical Care 2012, 16:459. 